\title{
Leichte Sprache im Polnischen. Überlegungen zu einem neuen sprachlichen Phänomen
}

\author{
Easy-to-read Polish. Considerations \\ on a new linguistic phenomenon
}

Prof. Dr. Dr. h.c. Helmut W. Schaller zum 80. Geburtstag

\begin{abstract}
The present article deals with Easy-to-read Polish, a language variety which has been created for target groups with difficulties in reading and understanding written standard text. It is systematically simplified in both syntax and vocabulary. In order to describe Easy-to-read Polish, this article consults findings made in the last few years in examinations of Easy-to-read German. The analysis is based on texts in Easy-to-read Polish which were published by the organization "Inclusion Europe" on their homepage. With the help of these texts, characteristic features of the Easy-to-read language on various linguistic levels such as the system of symbols, morphology, syntax, vocabulary, semantics, and text are being worked out and illustrated by examples. It turns out that findings on Easy-to-read German are definitely helpful and inspiring for the elaboration of this language variety in Polish. Yet, in many cases more detailed language specific research is required.
\end{abstract}

KEYWORDS: Easy-to-read, accessible communication, Polish, linguistic analysis, Inclusion Europe.

SCHLÜSSELWÖRTER: Leichte Sprache, barrierefreie Kommunikation, linguistische Analyse, Inclusion Europe.

\section{EINFÜHRUNG}

In den letzten Jahrzehnten sind in vielen Ländern der Welt verstärkte Bemühungen um die rechtliche Gleichstellung und Inklusion von Menschen mit Behinderungen zu beobachten. Ein wichtiger Bereich ist dabei die barrierefreie Kommunikation. Zu dieser leistet die sogenannte Leichte Sprache (im Folgenden: LS) einen Beitrag, die sich in westlichen Ländern bereits etabliert hat, in Osteuropa im Vergleich dazu aber noch kaum bekannt ist. Sie richtet sich an Menschen, die aufgrund einer kognitiven oder sensorischen Behinderung, aber auch aus anderen Gründen Schwierigkeiten mit der Lektüre standardsprachlicher Texte haben, und ermöglicht ihnen den Zugang zu Informationen und damit die Teilhabe am 
gesellschaftlichen Leben (vgl. Radünzel 2018: 269). In diesem Artikel werden Grundprinzipien und Merkmale der LS thematisiert, wie sie in jüngster Zeit in sprachwissenschaftlichen Publikationen zum Deutschen beschrieben worden sind. Demgegenüber steht eine linguistische Fundierung der entsprechenden Varietät im Polnischen noch aus. In einem früheren Aufsatz (Radünzel 2017) wurden polnischsprachige LS-Texte vorgestellt und mit ihren deutschen Pendants verglichen. Des Weiteren wurde das Konzept der LS in seiner Genese und mit seinen typischen Merkmalen auf dem 16. Internationalen Slavistenkongress in Belgrad präsentiert und anhand von russischen, polnischen und serbischen Beispielen verdeutlicht (vgl. dazu den Aufsatz Radünzel 2018). Hieran schließt sich der vorliegende Beitrag an, der die Intention verfolgt, die Umsetzung des Konzepts der LS im Polnischen auf der Grundlage weiterer Texte nochmals gezielt zu untersuchen, Lösungsvorschläge zu unterbreiten, dabei Erkenntnisse aus den germanistischen Arbeiten nutzbar zu machen und zu zeigen, wo Übertragungen möglich und wo gesonderte Forschungen zu den Spezifika des Polnischen nötig sind. Dass dabei stellenweise mehr Fragen aufgeworfen als Antworten gegeben werden, ist der Tatsache geschuldet, dass es sich um erste Überlegungen handelt und keine empirischen Studien mit Referenzpersonen durchgeführt wurden (ebenso Radünzel 2018: 269).

\section{LEICHTE SPRACHE - GRUNDLEGENDE AUSFÜHRUNGEN UND DIDAKTISCHE FRAGESTELLUNGEN}

Die LS kann kurz als eine Varietät einer beliebigen Ethnosprache charakterisiert werden, die sowohl im Bereich des Satzbaus als auch des Wortschatzes systematisch reduziert ist und sich durch eine systematische „Reduktion mit Bezug auf das Weltwissen, das für die Lektüre vorausgesetzt wird“, sowie durch eine „besondere Form der visuellen Aufbereitung" auszeichnet (Maaß 2015: 11f.; zu grundlegenden Merkmalen der LS bereits Radünzel 2017: 52-59, 2018: 272f.). Sie ist Bestandteil des Systems einer Sprache und lässt sich auf allen Ebenen linguistisch beschreiben (Bredel \& Maaß 2016: 14, 80). Zu den Adressaten der LS gehören die folgenden Gruppen (siehe auch Radünzel 2017: 55-57, 2018: 273f.): Menschen mit geistiger Behinderung, Lernschwierigkeiten, Demenz (die als erworbene geistige Behinderung betrachtet wird) oder Aphasie (einer Sprachstörung, die nach vollzogenem Spracherwerb auftritt), prälingual Gehörlose, d.h. Personen, die gehörlos geboren wurden oder die ihr Gehör noch vor abgeschlossenem Spracherwerb verloren haben, funktionale Analphabeten, d.h. Menschen, die zwar über eine Schulausbildung verfügen, aber dennoch das sinnentnehmende Lesen von standardsprachlichen Texten nicht beherrschen, Migranten bzw. Personen, die eine Zweitsprache erlernen. 
Die internationale Entwicklung der LS inklusive des Beitrags wesentlicher Akteure ist an anderer Stelle bereits ausführlich dargestellt worden (vgl. Radünzel 2017: 51f., 2018: 271). Hierbei wurde auf die Rolle von Selbstvertretungsorganisationen und Initiativen wie der „Europäischen Vereinigung der International League of Societies for Persons with Mental Handicap" (ILSMH), die sich später in „Inclusion Europe“ umbenannte (vgl. die Internetseite http:/ /inclusion-europe.eu), und dem deutschen "Netzwerk Leichte Sprache“ (www.leichtesprache. org) hingewiesen. Diese verfassten nicht nur Texte in LS, sondern entwickelten aus ihrer praktischen Erfahrung heraus Richtlinien, die ihrerseits von anderen Stellen aufgegriffen wurden. So bildete das Regelwerk des Netzwerks Leichte Sprache (im Folgenden zitiert als NLS) 2013 die Grundlage für eine Broschüre des Bundesministeriums für Arbeit und Soziales zu diesem Thema (im Folgenden: BMAS). Darüber hinaus trug die Aktivität dieser Vereinigungen dazu bei, dass das Konzept der LS nicht nur größere Bekanntheit in der Öffentlichkeit erlangte, sondern auch gesetzlich verankert wurde. Dies erfolgte in Deutschland durch die Barrierefreie-Informationstechnik-Verordnung (aktuelle Fassung BITV 2.0 von 2011, im Folgenden zitiert als BITV) und die neue Fassung des Behindertengleichstellungsgesetzes (BGG) von 2016 (dazu bereits Radünzel 2017: 49f., 2018: 270f.). Von internationaler Bedeutung ist die UN-Konvention über die Rechte von Menschen mit Behinderungen (2006), gemäß der die Vertragsstaaten geeignete Maßnahmen treffen, „um den Zugang von Menschen mit Behinderungen zu den neuen Informations- und Kommunikationstechnologien und -systemen, einschließlich des Internets, zu fördern“ (Artikel 9) (vgl. Radünzel 2017: 49, 2018: 269). Diese Konvention wurde von vielen Staaten ratifiziert, darunter Polen und Deutschland.

Deutlich später als die Ausarbeitung der LS durch Praxisvertreter setzte die sprachwissenschaftliche Beschäftigung mit dieser Varietät ein. In der Germanistik lässt sich mittlerweile eine rege Forschungstätigkeit zu diesem Thema beobachten, was für die Slawistik nicht in gleicher Weise gilt. Jedoch wurden auch hier erste Studien vorgelegt (zum Forschungsstand Radünzel 2017: 52, 2018: 272).

Für Polen kann man feststellen, dass das Konzept der LS noch am Anfang seiner Entwicklung steht und außerhalb von Organisationen für Menschen mit geistiger Behinderung kaum bekannt ist (so Przybyła-Wilkin 2016: 135, 139f.). Eine Überprüfung offizieller Internetseiten polnischer politischer Institutionen ergab, dass weder der Präsident (www.prezydent.pl) noch der Ministerpräsident und die Regierung (www.premier.gov.pl) oder das Parlament (www.sejm.pl) Informationen in LS bereitstellen (zuletzt geprüft am 3.11.2019) ${ }^{1}$. Anzumerken ist, dass die Seite des Ministerpräsidenten und der Regierung einen Button

\footnotetext{
${ }^{1}$ Dies ließ sich ebenso für die Russische Föderation, Tschechien, Serbien und Kroatien konstatieren (Radünzel 2018: 271).
} 
„Dostępnośćc enthält, über den man Informationen für behinderte Benutzerinnen und Benutzer aufrufen kann. Dabei ist auch die Rede davon, Informationen verständlich für jedermann gestalten zu wollen: „Pamiętamy, że z dostępności korzystają tak naprawdę wszyscy. Dlatego dbamy również o: Zrozumiałość - staramy się wyjaśniać trudne słowa, rozwijać skróty oraz unikać niezrozumiałego słownictwa. Czytelność - staramy się odpowiednio formatować teksty, w tym stosować wypunktowania, wyróżnienia i możliwie krótkie akapity.“ (fett im Original). Man ist sich des Problems also offensichtlich bewusst, hat jedoch bislang noch nicht den Schritt vollzogen, Texte in LS anzubieten. LS-Versionen von Informationsmaterialien auf Polnisch wurden u.a. von der Organisation "Inclusion Europe" erstellt, auf deren Bedeutung für die Entwicklung des LS-Konzepts in den früheren Arbeiten hingewiesen wurde. Aufgrund der Tatsache, dass Polen die UN-Behindertenrechtskonvention ratifiziert hat und sich darüber hinaus als EU-Mitglied an internationalen Entwicklungen orientiert, kann man davon ausgehen, dass sich die LS künftig auch in Polen stärker verbreiten wird.

Die LS ist in vielfacher Hinsicht mit sprach- und fachdidaktischen Fragestellungen verknüpft. Sie wird in der Förderschule und im inklusiven Unterricht eingesetzt (vgl. Rink 2014; Maaß 2015: 91), wobei der letztgenannte Bereich aufgrund der Gesetzeslage in Deutschland in der Zukunft eine immer größere Rolle spielen wird. Zudem hat sich der Adressatenkreis der LS ausgeweitet. Nachdem diese Varietät ursprünglich für überschaubare Zielgruppen wie Menschen mit Lernschwierigkeiten bzw. geistiger Behinderung oder prälingual Gehörlose entwickelt worden war, werden neuerdings u.a. auch Migranten bzw. Menschen, die Deutsch (oder eine andere Ethnosprache) als Zweitsprache erlernen, zu den potentiellen Adressaten gerechnet (BMAS: 5, 9, 16; Inclusion Europe (im Folgenden: IE): 6; NLS: 1; Maaß 2015: 18; Maaß, Rink \& Zehrer 2014: 58; Bredel \& Maaß 2016: 169-172; Bock 2014: 19; Gross 2015: 87; Oomen-Welke 2015: 28-30; dazu schon Radünzel 2017: 57). Diese Gruppe kann angesichts der aktuellen politischen Lage nicht vernachlässigt werden, da im Zuge der Flüchtlingskrise viele Menschen aus einer Notsituation heraus nach Deutschland gekommen sind, die keinerlei Möglichkeit hatten, vorher Sprachkenntnisse zu erwerben, um sich hier in die Gesellschaft, darunter auch in den Arbeitsmarkt, integrieren zu können. Verschiedene Autoren halten es für vorstellbar, die LS im Bereich Deutsch als Fremdsprache zu nutzen, und betrachten sie als ein mögliches „Durchgangsstadium“ hin zur Lektüre standardsprachlicher Texte (z.B. Maaß, Rink \& Zehrer 2014: 58; Bredel \& Maaß 2016: 144; kritisch dazu Zurstrassen 2017: 63f.).

Diese Entwicklungen haben es mit sich gebracht, dass die deutsche LS in verschiedenen jüngeren Publikationen gezielt aus einer sprachdidaktischen Perspektive betrachtet bzw. kritisch diskutiert wird. Es sei hier exemplarisch auf ausgewählte Arbeiten verwiesen, um einige grundlegende Probleme zu 
verdeutlichen, die in gleicher Weise auch im Zusammenhang mit anderen Ethnosprachen relevant sind. So stellt Kilian (2017) die Frage nach der sprach-bzw. fachdidaktischen Wertigkeit des Konzepts der LS. Mit „sprachdidaktischer Wertigkeit" meint er, „ob und inwiefern dieses Konzept einen effizienten und effektiven Beitrag zum sprachlichen Lernen und / oder zur sprachlichen Bildung (einschließlich des Erwerbs sprachlich gebundenen Wissens) zu leisten vermag" (Kilian 2017: 189). Der Autor untersucht den Bereich des Wortschatzes bzw. der (lexikalischen) Semantik, da der Aufbau des Fachwortschatzes der Unterrichtsfächer und der damit verbundenen kognitiven Konzepte ein zentrales fachdidaktisches Ziel darstellt. Kilian erörtert die Frage, ob LS als eine „Lernvarietät“ oder sogar als eine "Zielvarietät" im Rahmen des sprachlichen Lernens konzipiert werden kann. Dem Ansatz von LS als Zielvarietät erteilt er eine klare Absage, da hierfür keinerlei sprachdidaktisch vertretbare Begründung erkennbar sei (Kilian 2017: 192f.). Für den Ansatz als Lernvarietät sei die sprach- und fachdidaktische Effektivität und Effizienz der LS zu prüfen (Kilian 2017: 193). Nach Ansicht des Autors ist die Erweiterung der Adressatengruppe um Menschen mit spezifischen Lernschwierigkeiten im Kompetenzbereich des Lesens aus sprachdidaktischer Perspektive nicht vertretbar, da das Konzept der LS selbst keine Entwicklungsperspektive formuliere, eine Didaktik ohne Entwicklungsperspektive aber keine sei (Kilian 2017: 198). In der Folge thematisiert Kilian die Überlegung, ob die LS im inklusiven Unterricht ein didaktisches Instrument sein könne, um Schülerinnen und Schülern das Erreichen von Bildungsstandards zu ermöglichen bzw. zu erleichtern, wozu er sich skeptisch äußert (Kilian 2017: 202f.). Abschließend urteilt der Autor, dass das Konzept der LS „etwas vorschnell“ vom ursprünglichen Adressatenkreis (Menschen mit Behinderungen, Demenz oder Aphasie) auf Menschen mit Schwierigkeiten beim Auf- und Ausbau einer Lesekompetenz (z.B. funktionale Analphabeten, DaZ-Lernende, Lernende mit Schwierigkeiten beim Verstehen von Fach- und literarischen Texten) übertragen wurde. Bezüglich der Kompetenzbereiche Wortschatz und (lexikalische) Semantik stellt er fest, dass die bisherige Ausarbeitung der LS ,weder linguistischen (lexikologischen) noch sprach- und fachdidaktischen Anforderungen (in Bezug auf die lexikalisch-semantische Kompetenz)" genüge (Kilian 2017: 205). Des Weiteren resümiert Kilian, dass die LS „,weder im erstsprachlichen noch im zweitsprachlichen Unterricht als Ansatz einer sprachdidaktischen Konzeption zur Sprachförderung begriffen werden kann“ (Kilian 2017: 205).

Die didaktische Bedeutung der LS im inklusiven Unterricht (z.B. in den Bereichen Literatur und Geschichte) behandeln Riegert und Musenberg (2017). Aufgrund der Notwendigkeit der Binnendifferenzierung in einem (zieldifferenten) Fachunterricht beurteilen sie den Einsatz der LS als ,grundsätzlich hilfreich zur Überwindung von Lernbarrieren" (Riegert \& Musenberg 2017: 396). Sie zeigen 
Spezifika des Literatur- und des Geschichtsunterrichts auf, die besondere Anforderungen an die LS in diesen Bereichen bedingen, und formulieren Fragen für die Theoriebildung, die empirische Forschung und die Unterrichtsgestaltung.

Überlegungen aus der Perspektive des Faches Deutsch als Fremdsprache bzw. Deutsch als Zweitsprache stellt Heine (2017) an. Sie wirft die Frage nach einem möglichen Zusammenhang zwischen LS-Texten und Texten für Fremdsprachenlernende auf und analysiert die vorliegenden Regeln für LS im Hinblick darauf, ob sie auf Menschen mit einer anderen Muttersprache übertragbar bzw. aus dem Kontext des Sprachunterrichts bekannt sind. Anschließend überprüft sie den gewissermaßen umgekehrten Fall, nämlich die Frage, ob Lehrbuchtexte für L2-Lernende als LS-Texte verwendet werden können. Hierbei stellt Heine zunächst mit Recht fest, dass viele der existierenden LS-Regeln für L2-Lernende irrelevant oder sogar kontraproduktiv sind, z.B. die Vermeidung von Jahreszahlen, hohen Zahlen, Prozentzahlen oder Sonderzeichen (Heine 2017: 406f.). Auch Empfehlungen wie der Verzicht auf Fach- und Fremdwörter (bei denen es sich häufig um Internationalismen handelt) oder die Bevorzugung von Verben gegenüber Substantiven können für L2-Lernende nicht übernommen werden (Heine 2017: 407f.). Aus ihrer Analyse ausgewählter Regeln schlussfolgert die Autorin, dass LS-Texte nicht für die Zielgruppe der L2-Lernenden geeignet sind (Heine 2017: 410). Umgekehrt entsprechen aber auch Lehrbuchtexte aus dem DaF- bzw. DaZ-Unterricht selbst auf einem verhältnismäßig niedrigen Niveau nicht den Regeln der LS und können für deren Zielgruppen nicht genutzt werden (Heine 2017: 412).

Es liegt auf der Hand, dass die exemplarisch vorgestellten didaktischen Überlegungen zum Einsatz der LS nicht ausschließlich an die deutsche Ethnosprache bzw. an die Verhältnisse in Deutschland gebunden sind. Sie wären in gleicher Weise für die entsprechende polnischsprachige Varietät und ihre Verwendung im inklusiven Schulunterricht in Polen bzw. im Unterricht für Migranten, die das Polnische als Zweit- bzw. Fremdsprache erlernen, zu berücksichtigen. Jedoch ist hier der Schwerpunkt zunächst auf die sprachwissenschaftlich fundierte Ausarbeitung einer solchen Varietät zu setzen, die in Deutschland bereits stärker vorangeschritten ist.

\section{AUSGEWÄHLTE MERKMALE DER LEICHTEN SPRACHE IM POLNISCHEN - DARGESTELLT AM BEISPIEL VON TEXTEN}

Die LS zeichnet sich grundsätzlich auf allen sprachlichen Ebenen durch Unterschiede zur Standardsprache aus. Für das Deutsche ist dies besonders in Monographien von Maaß und Bredel (Maaß 2015; Bredel \& Maaß 2016) überzeu- 
gend nachgewiesen worden. An anderer Stelle (Radünzel 2017) wurden bereits Besonderheiten polnischer LS-Texte im Vergleich zu deutschen Versionen herausgearbeitet. Dies erfolgte anhand von Beispielen, die Texten der Organisation "Inclusion Europe“ zur Arbeit der Vereinten Nationen und zur Zugänglichkeit von Wahlen in Europa entstammten. Ferner wurde von mehreren Autoren der Versuch unternommen, Erkenntnisse der Germanistik und Vergleiche von LS-Texten in verschiedenen Sprachen für die Ausarbeitung einer solchen Varietät in den slawischen Sprachen auf linguistischer Basis nutzbar zu machen (Usova 2015; Przybyła-Wilkin 2016; Usova 2017; Just 2017; Radünzel 2017; Radünzel 2018). In der Folge sollen die Ansätze zu einer sprachwissenschaftlichen Beschreibung der LS im Polnischen noch einmal komprimiert dargestellt bzw. ausgebaut werden. Hierbei ergab sich jedoch ein Problem, weiteres analysefähiges Material zu finden. Als die oben erwähnten Texte im November 2014 von der Homepage der Organisation „Inclusion Europe“ heruntergeladen wurden, waren dort noch mehrere Broschüren in polnischer LS zu anderen Themen vorhanden. Inzwischen wurde die Seite der Organisation stark überarbeitet und diese Materialien sind nicht mehr zu finden. Anfragen bei „Inclusion Europe“ nach polnischen LS-Texten (gestellt per E-Mail am 10.9.2016 und am 3.10.2016) blieben unbeantwortet, obwohl der Verein in seinem Internetauftritt ausdrücklich auf die Möglichkeit aufmerksam macht, ihn zu kontaktieren. Informationen über die Organisation und ihre Arbeit waren jedoch noch später in polnischer Sprache vorhanden (Zugriff am: 8.10.2016) und dienen als Grundlage für die nachstehenden Ausführungen. Bei Bedarf werden zudem nochmals die oben erwähnten Materialien über die Vereinten Nationen und die Wahlen herangezogen. Zunächst sei einiges zu den Texten auf der Homepage ausgeführt.

Die polnischsprachigen Informationen auf der Internetseite gliedern sich in die folgenden Rubriken: „O nas“ (Textumfang 281 Wörter), ,"Rzecznictwo wlasne (sic!)“ (234 Wörter), „Prawa“ (436 Wörter), „Polityki“ (427 Wörter), „Projekty“ (253 Wörter) und "Zaangazuj sie“ (356 Wörter). Diese Informationen sind nicht ausdrücklich (z.B. durch das entsprechende Siegel von „Inclusion Europe“) als Texte in LS gekennzeichnet. Auch fehlt die obligatorische Bebilderung. Sie richten sich jedoch zum einen an die ursprüngliche Zielgruppe der LS (Menschen mit geistiger Behinderung bzw. mit Lernschwierigkeiten), da man davon ausgehen kann, dass die Organisation bei der Gestaltung ihres Informationsangebots die Personen im Blick hat, die sie (potentiell) vertritt. Zum anderen weisen die Texte in ihrer graphischen und sprachlichen Gestaltung wesentliche Merkmale der LS auf und sind durchaus mit den Materialien über die UNO und die Wahlen vergleichbar, die explizit als LS-Versionen markiert sind. Zu diesen Merkmalen gehören die große Schrift, der Flattersatz, die starke Gliederung durch Überschriften, Zwischenüberschriften und Unterpunkte, das Fehlen 
von Silbentrennungen und die Tatsache, dass jeder Satz in der Regel auf einer neuen Zeile beginnt. Ausnahmen davon finden sich in den Texten "Projekty“ und "Zaangazuj sie“, wobei es sich vermutlich eher um Fehler handelt als dass dem eine Absicht zugrunde liegt. Besonders auffällig ist, dass die Texte mit den Buchstaben des für das Englische gültigen Alphabets geschrieben sind und mit Ausnahme des „'ó" keine der für das Polnische typischen Buchstaben (ą, ć, ę, l, ń, ś, ź, ż) enthalten. Sie widersprechen damit in eklatanter Weise dem Grundsatz, dass LS nicht „falsch" sein dürfe (vgl. „Falsches Deutsch vermeiden“ - Maaß 2015: 81-84, 179). Es ist nicht nachvollziehbar, warum die Texte so geschrieben wurden. Diese Schreibweise ist aus verschiedenen Gründen äußerst problematisch. Zum einen erschwert sie die Lektüre und verhindert den Wiedererkennungs- und Lerneffekt, da die Leser Wörter nicht wiedererkennen, die ihnen in standardsprachlichen Texten in ihrer orthographisch korrekten Form begegnen. Zum anderen dürfte sich die gesellschaftliche Akzeptanz der LS kaum erhöhen, wenn Textmaterial verfasst wird, das jeder des Polnischen Kundige auf den ersten Blick als fehlerhaft erkennt.

Aufgrund der schwierigen Quellenlage wurde jedoch entschieden, das Material trotzdem zu verwenden. Zudem ist anzumerken, dass auch deutsche LS-Texte oft von mangelhafter Qualität sind. Anhand der genannten Texte wird dargelegt, inwieweit ausgewählte für das Deutsche beschriebene Merkmale der LS auch hier nachweisbar sind, wo für das Polnische abweichende Daten vorliegen und was diese für eine Reglementierung der LS im Polnischen zur Folge haben. Hierbei geht es nicht darum, dem Polnischen Regelungen, die für eine andere Sprache getroffen wurden, aufzwingen zu wollen. Es versteht sich, dass eine Ausarbeitung dieser Varietät nur von muttersprachlichen Philologen in Zusammenarbeit mit Vertretern der Zielgruppen geleistet werden kann. Jedoch wird davon ausgegangen, dass die Forschung zur LS im Deutschen schon wichtige Erkenntnisse erbracht hat, die durchaus Anregungen für andere Ethnosprachen liefern können.

\subsection{Zeichensystem}

Im Bereich des Zeichensystems sind insbesondere die Interpunktionszeichen interessant (dazu bereits Radünzel 2017: 69-71). Hierbei werden im Deutschen Punkt, Fragezeichen, Ausrufezeichen, Doppelpunkt, Anführungszeichen, Klammern (zur Kommentierung), Divis (als Bindestrich) und Mediopunkt genutzt (zum Zeichensystem der LS ausführlich Bredel \& Maaß 2016: 221-296). Das Komma dagegen gehört nicht zum System der LS, da Sätze nur einzelne Aussagen enthalten sollen. Aufzählungen werden nicht in den Satz integriert, sondern 
als Listen mit Unterpunkten realisiert, Nebensätze werden in Hauptsätze umgewandelt (vgl. spätere Ausführungen zur Syntax). Häufig anzutreffen ist der Doppelpunkt. Er dient der Fokusbildung und der Einleitung von Aufzählungen, die ihrerseits die Form von Listen haben (Maaß 2015: 87; dazu auch Bredel \& Maaß 2016: 254f.). Anführungszeichen kennzeichnen Redeimporte und Passagen, die man nur erwähnt (Bredel \& Maaß 2016: 258f.; dazu auch Maaß 2015: 88). Der sogenannte Mediopunkt, ein Punkt auf halber Versalhöhe, wurde von der Forschungsstelle Leichte Sprache der Universität Hildesheim als Lesehilfe eingeführt (dazu schon Radünzel 2017: 69f., 2018: 276f.). Er grenzt die Bestandteile von Komposita, z.B. „Menschen • rechte“ oder "Gleichstellungs • gesetz", voneinander ab und erleichtert dadurch die Lektüre. Im Unterschied zur Abgrenzung der Bestandteile von Komposita mit Bindestrich, z.B. „Bundes-Tags-Wahl“ oder „Bundes-Kanzlerin“, wie sie aus der Praxis heraus entwickelte Ratgeber zur LS empfehlen (BITV: 20; BMAS: 26; IE: 23; NLS: 6), werden hiermit keine falschen Lernimpulse gesetzt. Da der Mediopunkt nicht so stark in die Wortgestalt eingreift, wird zudem die Wiedererkennung standardsprachlicher Wörter erhöht (Maaß, Rink \& Zehrer 2014: 62-64; Maaß 2015: 88-92; Bredel \& Maaß 2016: 331-338, 2017) ${ }^{2}$. Während Komposita im Deutschen sehr häufig vorkommen, mehr als zwei Stämme enthalten können und u.U. sehr lang und damit schwer zu lesen sind, liegt der Fall im Polnischen aufgrund der Wortbildungsspezifika anders. Es müsste geprüft werden, ob eine Gliederungshilfe für zusammengesetzte Wörter hier überhaupt nötig ist. Im Textkorpus wäre der Mediopunkt etwa bei „między •narodowy“ (Prawa) einsetzbar, eventuell auch bei den häufig auftretenden Komposita "nie• pełno • sprawny" und "nie•pełno•sprawność".

In den Texten auf der Homepage werden Punkt, Doppelpunkt, Ausrufezeichen und Klammern verwendet. Auch Anführungszeichen werden benutzt, z.B.: „Wspieramy naszych czlonków. / ,Inclusion Europe pomaga naszej organizacji, aby byla silniejsza." (O nas, kursiv im Original) ${ }^{3}$. Es scheint sich hier um ein Zitat bzw. um eine Äußerung einer unterstützten Organisation zu handeln, die durch die Anführungszeichen und den Kursivdruck doppelt hervorgehoben wird. Daneben markieren Anführungszeichen Bezeichnungen von Dokumenten oder anderen Rubriken der Internetseite und heben einmal eine Abkürzung hervor, z.B.: „Zapisz sie na biuletyn EPSA ,Europa dla nas!“" (Rzecznictwo wlasne), "Zobacz jak mozesz z nami pracowac ,Zaangazuj sie'." (O nas, ähnlich Prawa, Polityki, Projekty), „,W skrócie, czesto nazywa sie ja ,CPRD'.“ (Prawa). Entgegen

${ }^{2}$ Bei Befragungen von Zielgruppenvertretern wurde eine generelle Akzeptanz für den Mediopunkt festgestellt (Lasch 2017: 283).

${ }^{3}$ Aus Platzgründen wird darauf verzichtet, jeden Satz auf einer neuen Zeile beginnen zu lassen, wie es in den Texten auf der Internetseite zumeist der Fall ist bzw. wie es als Grundregel der LS gilt. Die Zeilenumbrüche werden durch / gekennzeichnet. 
der für das Deutsche aufgestellten Regel kommen Kommata vor, zum einen in Aufzählungen, zum anderen in Nebensätzen, z.B.: „Nasi czlonkowie to krajowe, regionalne i lokalne organizacje osób z niepelnosprawnoscia intelektualna i ich rodzin.“ (O nas). Aufzählungen könnten durch Unterpunkte gegliedert werden: „Nasi członkowie to organizacje osób z niepełnosprawnością intelektualną i ich rodzin. / To są: / • organizacje krajowe / • organizacje regionalne / • organizacje lokalne." Ausführungen zur Umformulierung von Nebensätzen folgen beim Thema Syntax (3.3.).

Eine weitere Regel für das Zeichensystem besagt, dass Zahlen stets als Ziffern (nur arabische, nicht römische) zu schreiben sind (dazu bereits Radünzel 2017: 72). Für das Deutsche ergibt sich ein besonderes Problem daraus, dass zwischen dem Zahlwort "ein" und dem unbestimmten Artikel "ein" unterschieden werden muss, woraus unterschiedliche Schreibweisen „20 Hunde, 1 Katze" vs. „Ein Kind muss Spielzeug haben“ resultieren (Maaß 2015: 94; zu derartigen Problemen Bredel \& Maaß 2016: 237f., 365-368). Dies ist für das Polnische naturgemäß nicht relevant, da es sich um eine artikellose Sprache handelt. Man könnte daher generell die Schreibung von Zahlen als Ziffern empfehlen. Diskutiert werden müsste, wie mit den Numeralia zu verfahren ist, bei denen unterschiedliche Genusformen existieren: „jeden głos, jedna książka, jedno miasto“ oder „1 głos, 1 książka, 1 miasto “, , dwa razy, dwie kobiety“ oder "2 razy, 2 kobiety“? In den Internettexten kommen lediglich Jahreszahlen vor. In den früher ausgewerteten LS-Texten über die Vereinten Nationen werden Zahlen teils als Ziffern, teils mit Worten geschrieben: „Wszystkie kraje członkowskie mają po 1 głosie w ONZ.“ (ONZ: 2), „Dwa razy do roku rządy spotykają się [...]" (ONZ: 7) (siehe Radünzel 2017: 72) ${ }^{4}$.

Beim Zeichensystem spielen zudem Fragen der typographischen Hervorhebung eine Rolle (dazu bereits Radünzel 2017: 66, 71, 88f.). Während z.B. Hervorhebungen durch Fettdruck sprachunabhängig empfehlenswert (und auch im Textmaterial belegt) sind, gäbe es für das Polnische die zusätzliche Möglichkeit, besonders wichtige Begriffe durch Großschreibung zu betonen. Es ist fraglich, ob dies sinnvoll ist oder eher zu einer Verwechslung mit Eigennamen führen würde. In den Texten findet man Großschreibung bei Eigennamen wie „Europejskie Stowarzyszenie Osób z Niepelnosprawnoscia Intelektualna i Ich Rodzin“" (O nas), „Europejska Platforma Rzeczników Wlasnych Praw“ (Rzecznictwo wlasne) oder "Organizacja Narodów Zjednoczonych" (Prawa). Diese Art der Hervorhebung wird zudem bei Begriffen wie „Prawa Czlowieka“ (Prawa), ,Integracja Spoleczna“ (Prawa, Polityki) oder „Edukacja Integracyjna“ (Prawa, Polityki) benutzt. In

${ }^{4}$ Alle Hervorhebungen durch Kursivdruck in Zitaten stammen - sofern nicht anders angegeben - von mir - C. R. 
dem Beispiel „Organizujemy Spotkania dla róznych grup“ (Polityki) ist unklar, ob die Großschreibung hier tatsächlich sinnvoll ist. Zudem ist Kursivdruck zu beobachten (O nas, Rzecznictwo wlasne, Prawa, Polityki, Projekty, Zaangazuj sie), der jedoch in vielen Ratgebern, darunter von "Inclusion Europe“ selbst (BMAS: 63; IE: 14; Europäische Vereinigung der ILSMH (im Folgenden: ILSMH): 17; NLS: 29), abgelehnt wird.

Aufgrund der oben angesprochenen Schreibung der Texte, die auch aus den zitierten Beispielen deutlich wird, sollte eine Vorgabe gemacht werden, das komplette Alphabet des Polnischen in seiner standardsprachlich korrekten Form zu benutzen (d.h. inklusive aller Buchstaben, die vom Englischen abweichen).

\subsection{Morphologie}

Im Bereich der Nominalflexion stellt sich die Frage nach der Anzahl der Kasus (vgl. auch Radünzel 2017: 75f., 2018: 274). Ein auffälliger Unterschied der deutschen LS zur Standardsprache besteht darin, dass sie ein Drei-KasusSystem aufweist. Es fehlt der Genitiv, der auch in der Standardsprache auf dem Rückzug ist und für den in der LS - je nach seiner konkreten Funktion - verschiedene Ersatzstrategien vorgeschlagen werden (Bredel \& Maaß 2016: 302-311). Im Unterschied dazu ist für das Polnische zum einen fraglich, ob der Genitiv für die Zielgruppen überhaupt eine Verständnishürde darstellt. Dies könnte nur durch empirische Studien ermittelt werden. Zum anderen ist der Genitiv wohl nicht verzichtbar, wenn man seine Funktionen bedenkt und die Anforderung, dass LS die grammatischen Regeln der Standardsprache nicht verletzen darf (zum Genitiv im Russischen Usova 2015: 68; Just 2017: 18-28; Usova 2017: 459; zum Russischen, Polnischen und Serbischen Radünzel 2018: 274f.). Der Genitiv kommt häufig nach Präpositionen vor, z.B. „bez", ,dla“, , do" oder "od", ohne die man schwerlich auskäme. Er steht außerdem nach Numeralia, z.B. „,siedem krajów“, oder Adverbien wie „wiele“, „dużo“ usw., z.B.: „Unia Europejska tworzy wiele europejskich praw." (Prawa), "Inclusion Europe publikuje duzo tekstów." (Zaangazuj sie). Auch bestimmte Verben verlangen den Genitiv, z.B.: „Dlatego potrzebujemy tez twojego wsparcia!“ (O nas), "Chcemy lepszego zycia dla osób z niepelnosprawnoscia intelektualna i ich rodzin w calej Europie: [...]" (Prawa). Des Weiteren ist der Genitiv der Verneinung zu berücksichtigen, z.B.: "Ale te pieniadze nigdy nie pokrywaja wszystkich potrzebnych kosztów." (Projekty). Dativ, Akkusativ und Lokativ sind offenbar ebenso unverzichtbar. Beim Instrumental wäre in einigen Fällen zu überlegen, ob er durch alternative Konstruktionen ersetzt werden kann (dazu bereits Radünzel 2017: 75f.; zum Russischen Radünzel 2018: 275). Ein Beispiel ist sein Vorkommen als Nominalprädikat nach „"być“ , z.B. 
"ONZ jest organizacją międzynarodową". Es ist wahrscheinlich, dass die Formulierung mit "to" als Kopula und Nominativ hier besser verständlich wäre, z.B. "ONZ to organizacja międzynarodowa". Im Textkorpus sind Formulierungen mit "to" und Nominativ nachweisbar, z.B.: „Inclusion Europe to stowarzyszenie osób z niepelnosprawnoscia intelektualna i ich rodzin w Europie." (O nas). Es kommt aber auch "być" + Instrumental vor, was zumindest teilweise umformuliert werden könnte, z.B.: „Osoby z niepelnosprawnoscia intelektualna sa obywatelami jak wszyscy inni.“ (Prawa) $\rightarrow$ "Osoby z niepełnosprawnością intelektualną to obywatele jak wszyscy inni.“, "Jednym z najwazniejszych dokumentów dla osób niepelnosprawnych jest Konwencja Praw Osób Niepelnosprawnych. / Ten dokument to miedzynarodowe prawo.“ (Prawa) $\rightarrow$ „Konwencja Praw Osób Niepełnosprawnych to bardzo ważny dokument dla osób niepełnosprawnych. / Ten dokument to międzynarodowe prawo." An anderer Stelle gestaltet sich eine Umformulierung schwieriger, z.B.: „Dowiedz sie czemu dobrze jest byc czescia EPSA.“ (Rzecznictwo wlasne), „Organizacje pracujace na poziomie krajowym moga byc pelnymi czlonkami." (Zaangazuj sie).

Für die Verbalflexion in der deutschen LS gilt, dass die Kategorien Person und Numerus ohne Einschränkung gebraucht werden können, dies für Genus Verbi, Modus und Tempus dagegen nicht zutrifft (nur Aktiv, Indikativ, Präsens und Perfekt) ${ }^{5}$ (Bredel \& Maaß 2016: 312; dazu schon Radünzel 2017: 74f.). Die uneingeschränkte Verwendbarkeit von Person und Numerus lässt sich auf das Polnische übertragen. Das Passivverbot folgt aus der Forderung, dass in LS-Texten möglichst an jeder Stelle klar sein muss, wer etwas tut bzw. zu tun hat. Die Umformung von Passiv- in Aktivkonstruktionen bringt das Problem mit sich, dass hier in aller Regel ein Handlungsträger angegeben werden muss, der nicht immer leicht oder eindeutig zu bestimmen ist. Im Textmaterial kommt das Passiv verschiedentlich vor und lässt sich je nach Zusammenhang unterschiedlich leicht ersetzen, z.B.: „Krajowe prawa tworzone sa przez kazde panstwo.“ (Prawa) $\rightarrow$ „Każde państwo tworzy krajowe prawa.“ , "Te pieniadze wydaje sie na rózne koszty [...]“ (Projekty) $\rightarrow$ „Inclusion Europe wydaje te pieniądze na różne koszty [...]“, „Maja prawo byc wlaczeni do wszystkich obszarów zycia." (Prawa) $\rightarrow$ eventuell „Osoby z niepełnosprawnością intelektualną mają prawo do włączenia do wszystkich obszarów życia." (wenngleich dieSubstantivierung hier auch nicht günstig ist).

Im Polnischen stellt sich die Frage nach dem Umgang mit der unpersönlichen "-no / -to"-Konstruktion sowie mit unpersönlichen Ausdrücken wie „wolno“ oder "można“, die weit verbreitet sind (dazu bereits Radünzel 2017: 74f.), z.B.:

${ }^{5}$ Die fehlende Lizensierung sprachlicher Mittel wie Konjunktiv oder Futur erfordert umfangreiche Überlegungen dazu, wie Kategorien wie Zukünftiges, Irreales usw. auf andere Weise ausgedrückt werden können (dazu ausführlich Bredel \& Maaß 2016: 431-469). 
„Wyniki projektów mozna zobaczyc na naszej stronie internetowej.” (Projekty). In dem zitierten Satz wäre es möglich, den Leser direkt anzusprechen, was in der LS generell empfohlen wird: „Możesz zobaczyć wyniki projektów na naszej stronie internetowej. " "Wolno“ kommt im Textmaterial nicht vor, jedoch in dem früher untersuchten Korpus: „Nie wolno także przeprowadzać eksperymentów medycznych na ludziach.“ (ONZ: 6). Wie bereits an anderer Stelle erläutert wurde, wäre für eine Umformulierung die Ermittlung eines Handlungsträgers obligatorisch. Eine Möglichkeit bestünde in der Aussage, dass Menschen keine medizinischen Experimente an anderen Menschen vornehmen dürfen, etwa „Ludzie nie mają prawa przeprowadzać eksperymentów medycznych na innych ludziach.“ (Radünzel 2017: 74f.). Die „-no / -to“ -Konstruktion ist auf der Homepage ebenfalls nicht belegt, dafür einmal in der Broschüre über die UNO (siehe Radünzel 2017: 74): „Założono ją w 1945 roku.“ (ONZ: 1). Dort wurde die folgende Umformulierung mit Angabe des Handlungsträgers vorgeschlagen: „51 krajów założyło Organizację Narodów Zjednoczonych w 1945 roku.“

Für den Konjunktiv müsste überprüft werden, ob es Fälle gibt, in denen er im Polnischen unverzichtbar ist. Dies gilt ebenso für das Futur, das in der deutschen LS nicht verwendet wird, obwohl es mit dem Hilfsverb "werden" einen eigenen und durchaus gut zu erkennenden Träger der grammatischen Funktion aufweist. Von den deutschen Vergangenheitstempora ist nur das Perfekt lizensiert (mit Ausnahme der Hilfsverben "sein" und "haben“ und der Modalverben „,können“, ,, sollen“, ,"wollen“, ,"müssen“, ,"mögen“ und „dürfen“, die ins Präteritum gesetzt werden dürfen) (Maaß 2015: 101; Bredel \& Maaß 2016: 323-326). Für das Polnische gilt die bereits andernorts (Radünzel 2017: 80, 2018: 275) getroffene Feststellung, dass sich ein Diskussionsbedarf erübrigt, da nur ein Vergangenheitstempus zur Verfügung steht, z.B.: „W Inclusion Europe przeprowadzilismy juz wiele projektów." (Projekty).

Der Imperativ wird in den Werken zur deutschen LS nicht angesprochen (dazu schon Radünzel 2017: 79). In bestimmten Textsorten müssen Aufforderungen an den Leser wie „Gehen Sie schnell zum Ausgang!“ o. Ä. jedoch unbedingt realisiert werden können. Erkennungszeichen sind das Ausrufezeichen, die Anredeform "Sie“ und die Spitzenstellung des Verbs, die vermutlich ausreichen, um Verständnisschwierigkeiten bei den Zielgruppen zu vermeiden. Anders liegt der Fall beim Imperativ der 2. Person Singular, wo teilweise Stammveränderungen auftreten, z.B. „Nimm ein Tier als Beispiel!“ Solche Formulierungen sind beispielsweise für Schulbücher für den inklusiven Unterricht in Jahrgangsstufen angemessen, in denen man die Schüler üblicherweise duzt. Auch hier wird die Aufforderung durch das Ausrufezeichen und die Spitzenstellung des Verbs gekennzeichnet. Ob die Stammveränderung in der Verbform Verständnisprobleme mit sich bringt, wäre gesondert zu untersuchen. Mit dem polnischen Imperativ 
verhält es sich im Prinzip ähnlich; unregelmäßig sind hier beispielsweise Formen wie „bądź!“ oder „jedz!“. Zu möglichen Verständnishürden kann ohne Studien mit Zielgruppen keine Aussage getroffen werden. Im Polnischen ist ferner zu überlegen, wie Aufforderungen an eine Leserschaft, die man siezt, formuliert werden sollten. Darauf wird im Zusammenhang mit der angemessenen Leseransprache noch eingegangen (Abschnitt 3.6.).

Eine weitere für das Polnische wichtige Fragestellung ist die Verwendung der Verbalaspekte. Im Textkorpus dominiert klar der unvollendete Aspekt, was sich u.a. damit erklären lässt, dass als Tempus vor allem das Präsens vorkommt. Von den insgesamt 315 auftretenden Verbformen entfallen mehr als drei Viertel (249, d.h. 79\%) auf den unvollendeten Aspekt. Bei den 66 vollendeten Formen dominieren Imperative und Infinitive mit je 28 Belegen, z.B.: „Zapisz sie na biuletyn EPSA [...]" (Rzecznictwo wlasne), „Inclusion Europe i jej czlonkowie chca to zmienic!“ (Prawa). Des Weiteren kommen vier aktive Präterital- und eine Futurform im vollendeten Aspekt sowie fünf Partizipien Präteritum Passiv vor, z.B.: "Zaczelismy w 1988 roku.“ (O nas), „Na pewno znajdziesz wsród nich wydarzenia interesujace i dostepne dla Ciebie.“ (Zaangazuj sie), „[Ten dokument] Zostal stworzony przez Narody Zjednoczone dla wszystkich osób niepelnosprawnych na calym swiecie." (Prawa). Es ist zweifelhaft, ob die vollendeten Formen in den Beispielen gegen unvollendete ausgetauscht werden könnten, ohne dass es zu inakzeptablen Bedeutungsveränderungen bzw. zu einer Verletzung der Forderung nach grammatischer Korrektheit käme. Aufgrund der tiefen Verankerung der Kategorie des Aspekts im System des Polnischen und der anderen slawischen Sprachen und der verschiedenen Funktionen, die die Aspektformen erfüllen, ist nicht nur zu fragen, ob eine Reduzierung hier vorstellbar wäre, sondern auch zu überprüfen, ob Angehörige der LS-Zielgruppen überhaupt Schwierigkeiten damit haben.

\subsection{Syntax}

Eine augenfällige Besonderheit der LS-Syntax, die viele Formulierungsprobleme nach sich zieht, besteht darin, dass es keine zusammengesetzten Sätze geben soll. Für das Deutsche haben Bredel und Maaß eine Vielzahl von Vorschlägen zur Vermeidung von subordinierenden und koordinierenden Konstruktionen, vor allem zur Umformulierung von Nebensätzen, vorgelegt (Bredel \& Maaß 2016: 383-415; vorher schon Maaß 2015: 109-118; vgl. Vorschläge zum Polnischen in Radünzel 2017: 76-79, zum Russischen, Polnischen und Serbischen in Radünzel 2018: 275f.). Das analysierte Material weist entgegen der Regel viele Nebensätze auf. Anzumerken ist, dass pauschale Verbote bestimmter sprachlicher Mittel 
und Konstruktionen durchaus zur Diskussion gestellt werden sollten. So haben beispielsweise empirische Untersuchungen zur deutschen LS gezeigt, dass Zielgruppenvertreter einige Arten von Nebensätzen erfassen konnten (Lasch 2017: 294). Dennoch erscheint es gerechtfertigt, Vorschläge für eine Umformulierung auszuarbeiten, die später Gewährspersonen vorgelegt werden können, um deren Urteil zu ihrer Verständlichkeit und Akzeptabilität einzuholen. Im Folgenden werden aus Platzgründen nur die Nebensatztypen des Polnischen betrachtet, die im Korpus belegt sind.

(1) Finalsätze: Umformulierung mit „chcieć" + „dlatego“, z.B.: „Organizujemy konferencje, aby dzielic sie doswiadczeniami.“ (Rzecznictwo wlasne) $\rightarrow$ "Chcemy dzielić się doświadczeniami. / Dlatego organizujemy konferencje."

(2) Kausalsätze: Umformulierung mit "dlatego" oder "bowiem“, z.B.: "Jest tez wazna dla innych krajów na swiecie, poniewaz daje im dobry przyklad." (Polityki) $\rightarrow$ „Polityka europejska daje innym krajom na świecie dobry przykład. / Dlatego polityka europejska jest ważna dla tych krajów.“ oder „Polityka europejska jest też ważna dla innych krajów na świecie. / Polityka europejska daje bowiem tym krajom dobry przykład."

(3) Konditionalsätze: Umformulierung mit Frage + "to“" , z.B.: „Jezeli chcesz ofiarowac pieniadze, aby pomagac w naszej pracy podaruj teraz." (O nas, Zaangazuj sie) $\rightarrow$ „Chcesz pomagać w naszej pracy? / Chcesz ofiarować pieniądze? / To podaruj teraz!“ oder besser „Chcesz wspierać naszą pracę? / Chcesz ofiarować pieniądze? / To wpłać pieniądze teraz!"

(4) Relativsätze, z.B.: „Publikujemy wskazówki na temat spraw, którymi zajmuje sie rzecznictwo wlasne.“ (Rzecznictwo wlasne) $\rightarrow$ „Rzecznictwo własne zajmuje się różnymi sprawami. / Publikujemy wskazówki na temat tych spraw.", „Unia Europejska to jest grupa krajów, które pracuja razem.“ (Polityki) $\rightarrow$ „Unia Europejska to jest grupa krajów. / Te kraje pracują razem."

(5) Objektsätze: Umformulierung in Hauptsätze nach einem Doppelpunkt, z.B.: "Nasza konstytucja mówi kim jestesmy i jak pracujemy.“ (O nas) $\rightarrow$ "Nasza konstytucja mówi: / Kim jesteśmy. / Jak pracujemy.“, „Oznacza, ze wszystkie osoby z niepelnosprawnoscia intelektualna moga miec mozliwosc w pelni brac udzial w zyciu spolecznym." (Polityki) $\rightarrow$ „To oznacza: / Wszystkie osoby z niepełnosprawnością intelektualną mogą mieć możliwość w pełni uczestniczyć w życiu społecznym." oder kürzer „,[...] mogą uczestniczyć w życiu społecznym."

(6) weitere Nebensätze: beispielsweise Umformulierung in Hauptsätze, z.B.: "Oznacza, ze osoby z niepelnosprawnoscia intelektualna moga zadecydowac same o tym gdzie i z kim chca mieszkac.“ (Polityki) $\rightarrow$ „To oznacza: / Osoby z niepełnosprawnością intelektualną mogą zadecydować same: / Tu chcemy mieszkać. / Tak chcemy mieszkać.“ oder eventuell „[...] mogą zadecydować same o miejscu zamieszkania". 
Betreffs der Wortfolge favorisieren Bredel und Maaß grundsätzlich die Satzgliedstellung Subjekt - Prädikat - Objekt, aber nur dann, wenn dem nichts entgegensteht (Maaß 2015: 107f.; zur Wortstellung Bredel \& Maaß 2016: 415-425). Es wäre zu untersuchen, ob dies für das Polnische in gleicher Weise gilt.

Ein Spezifikum der polnischen Standardsprache liegt darin, dass ein Personalpronomen als Subjekt fehlen und die Person allein durch die Verbalendung ausgedrückt werden kann (im Unterschied zum Deutschen, wo die Subjektposition besetzt sein muss) (zu diesem Problem bereits Radünzel 2017: 83f.). Es ist zu fragen, ob dies in der polnischen LS beibehalten werden kann. Empirische Studien müssten zeigen, ob es für Vertreter der Zielgruppen schwierig ist, die handelnde Person ausschließlich an der Verbalendung zu erkennen. Unabhängig davon könnte darüber nachgedacht werden, die Personalpronomina zu setzen, da gemäß dem Redundanzprinzip (dazu Maaß 2015: 80f.) Informationen mehrfach gegeben bzw. gestützt werden sollen, um die Textrezeption zu erleichtern, z.B.: „Pracujemy nad róznymi projektami razem z naszymi czlonkami.“ (Polityki) $\rightarrow$ „My pracujemy nad różnymi projektami razem z naszymi członkami.“, , Mozesz zapisac sie na listy dyskusyjne na rózne tematy.“ (Zaangazuj sie) $\rightarrow$ „Ty możesz zapisać się na listy dyskusyjne na różne tematy."

\subsection{Lexik}

Im Bereich der Lexik steht man im Polnischen vor ähnlichen Problemen wie im Deutschen, so dass sich folglich auch die Übernahme von Lösungsansätzen anbietet. In beiden Sprachen ist die Forderung diverser Regelwerke, möglichst "einfache“ und "leicht verständliche“ Wörter zu verwenden (BITV: 20; BMAS: 22, 24; IE: 10; ILSMH: 12f.; NLS: 4), intuitiv nachvollziehbar, aber schwer umzusetzen, da hierfür klar sein müsste, wann genau ein Wort diese Bedingungen erfüllt (zur Lexik ausführlich Bredel \& Maaß 2016: 339-382, zu dieser Problematik bereits Radünzel 2017: 72, 2018: 276). Bredel und Maaß (2016: 347) setzen folgende Kriterien für die Auswahl von Wörtern in LS an: hohe Gebrauchsfrequenz, große diskursive Reichweite, Medienneutralität, denotative Präzision, konnotative Neutralität, stilistische Neutralität, keine Metaphorik, morphologische, graphematische und phonologische Einfachheit, früher Erwerbszeitpunkt, später Verlust. Wenn selten vorkommende Wörter in einem Text eine zentrale Rolle spielen, muss man sie einführen und ggf. erklären oder bildlich darstellen (Maaß 2015: 95f.).

Der Forderung, möglichst kurze Wörter zu benutzen, ist im Polnischen leichter nachzukommen als im Deutschen, da hier Komposita, zumal solche mit mehr als zwei Stämmen, deutlich seltener auftreten. Dies gilt ebenfalls für 
andere slawische Sprachen (Radünzel 2018: 276). Im Fall von komplexen Wörtern, auf die nicht verzichtet werden kann, ist der oben erwähnte Mediopunkt als Lesehilfe auch für das Polnische in Erwägung zu ziehen. Wiederum in beiden Sprachen ist es sinnvoll, Fach- und Fremdwörter nach Möglichkeit zu vermeiden oder sie zu erklären, sofern sie für einen Text wesentlich sind (Maaß 2015: 97f.; vgl. auch Bredel \& Maaß 2016: 347-355, dazu schon Radünzel 2017: 73, 2018: 276). In den Texten finden sich verschiedene Erklärungen wichtiger Begriffe, z.B.: „Edukacja Integracyjna / Oznacza, ze uczniowie z niepelnosprawnoscia intelektualna otrzymuja odpowiednie wsparcie, aby mogli chodzic / do takiej samej szkoly co wszyscy inni." (Polityki). Dies wäre nach den Regeln der LS freilich umzuformulieren, etwa wie folgt: „Edukacja Integracyjna: / To oznacza: / Uczniowie z niepełnosprawnością intelektualną mogą chodzić do takiej samej szkoły co wszyscy inni uczniowie. / Dlatego uczniowie z niepełnosprawnością intelektualną otrzymują wsparcie."

Im Fall von Abkürzungen, für die oft pauschal ein Verzicht empfohlen wird, ist eine Unterscheidung zwischen schriftbasierten Abkürzungen, die beim lauten Lesen aufgelöst werden (z.B. "usw."), und Siglenwörtern, die man so ausspricht (z.B. „WC“), vorzunehmen (zu Abkürzungen bereits Radünzel 2017: 73, 2018: 276). Maaß weist mit Recht darauf hin, dass man zwar Erstere, nicht aber Letztere sinnvoll vermeiden kann. Siglenwörter sind bekannter als die zugehörigen ausgeschriebenen Formen und begegnen jedem häufig im Alltag, was sie im Grunde unverzichtbar macht (Maas 2015: 98f.). Im Textmaterial werden Abkürzungen teilweise explizit eingeführt, teilweise stehen sie in unmittelbarer Nähe der Vollformen, ohne dass ein Bezug hergestellt wird, z.B.: „Na poziomie Europejskim jestesmy czlonkiem Europejskiego Forum Osób Niepelnosprawnych. / W skrócie nazywamy to forum EDF, od jego angielskiej nazwy. / EDF to Europejskie stowarzyszenie osób niepelnosprawnych.“ (O nas), "Organizacja Narodów Zjednoczonych tworzy prawa dla wszystkich na swiecie. / Inclusion International pracuje razem z ONZ [...]" (Prawa). Bredel und Maaß plädieren dafür, Erläuterungen in Leserichtung einzufügen, da die umgekehrte Reihenfolge Referenzpersonen Probleme bereitet habe (Maaß 2015: 138f.; Bredel \& Maaß 2016: 509-511).

\subsection{Semantik}

Aus dem Feld der Semantik seien hier zwei Phänomene herausgegriffen: Metaphern und Negation. Das generelle Metaphernverbot, das die nicht linguistisch fundierten Ratgeber festlegen (BMAS: 33; IE: 10; ILSMH: 14; NLS: 10), lässt sich nicht halten, da Metaphern von grundlegender Bedeutung für 
Sprache und Denken sind (zu dieser Frage bereits Radünzel 2017: 80f.). Jedoch ist auch klar, dass Metaphern wie „Rabeneltern“ Textrezipienten durchaus vor Verständnisprobleme stellen können. Bredel und Maaß (2016: 469-479) unterscheiden zwischen unvermeidlichen Metaphern, Metaphern, die das Verstehen erleichtern, und Metaphern, die den Verstehensprozess erschweren, und erläutern dies an Beispielen wie den folgenden. Die Metaphern der ersten Gruppe werden meist gar nicht als solche wahrgenommen und sind nicht ersetzbar, z.B. „Tischbein“ oder „Wasserhahn“. Die zweite Gruppe von Metaphern hilft dabei, sich bestimmten Konzepten zu nähern und sie anschaulich zu machen, z.B. die Aussage, dass Strom "fließt", oder der Begriff „Leichte Sprache“ selbst. Problematisch für die Adressaten der LS sind die Metaphern der letzten Gruppe, die folglich vermieden oder erklärt werden müssen, z.B. statt „Rabeneltern“ - „schlechte Eltern“ oder "Peter sagt: ,Ralf und Maria sind Raben • eltern.' Raben • eltern heißt: Ralf und Maria sind schlechte Eltern." Teilweise können Metaphern in Vergleiche aufgelöst werden. Hierdurch wird einerseits das Verständnis erleichtert, andererseits bleiben Bildhaftigkeit und Anschaulichkeit dennoch erhalten, z.B. „Er ist ein Löwe.“ $\rightarrow$ „Peter ist so stark wie ein Löwe." (Maaß 2015: 124f.; dazu auch Maaß, Rink \& Zehrer 2014: 65f.). In den Informationstexten von "Inclusion Europe“ werden keine Metaphern der dritten Gruppe benutzt.

Für die Negation gilt (ebenso wie für Metaphern), dass sie sich aufgrund ihrer zentralen Bedeutung für die Sprache selbst nicht vollkommen vermeiden lässt (vgl. die Forderungen in BITV: 20; BMAS: 32; IE: 11; ILSMH: 13; NLS: 10) (dazu Maaß 2015: 126-128; Bredel \& Maaß 2016: 460-469, siehe bereits Radünzel 2017: 65, 81). Im Deutschen gibt es die n- und die k-Negation (mit den Markern "nicht" bzw. „kein“), wovon die Erstgenannte vorzuziehen ist, da sie den besser erkennbaren Träger hat (besser "Wir haben heute nicht Kuchen gebacken." als "Wir haben heute keinen Kuchen gebacken.", Maaß 2015: 77, 127). Damit sie nicht übersehen werden, sind Negationsmarker in vielen LS-Texten fett gesetzt (so auch der entsprechende Vorschlag in Maaß 2015: 126-128, 182 sowie Bredel \& Maaß 2016: 468). Dies kann man auf das polnische „nie“ übertragen (im Textkorpus nicht gegeben), z.B.: „Chcemy praw nie przyslug!“ (Prawa), „Nie dostaja informacji w dostepnej formie.“ (Prawa) $\rightarrow$ "Osoby z niepełnosprawnością intelektualną nie dostają informacji w dostępnej formie." , "Nie dyskryminacja“ (Polityki). Im Fall der doppelten Verneinung könnten beide Marker hervorgehoben werden, z.B.: "Ale te pieniadze nigdy nie pokrywaja wszystkich potrzebnych kosztów." (Projekty). Eine neuere Studie (Bredel, Lang \& Maaß 2016) hat jedoch die These, dass sich der Fettdruck von Negationsmarkern positiv auf das Verständnis auswirkt, nicht bestätigen können. Hier sollten auch für das Polnische gesonderte Untersuchungen durchgeführt werden. 


\subsection{Textebene}

Aufgrund der starken Reduzierung des morphologischen, syntaktischen und lexikalischen Inventars kann in der LS vieles erst auf der Textebene ausgedrückt werden. Das Problem besteht darin, dass die Leser aber möglicherweise gar nicht zu dieser Ebene gelangen, da sie sie über der Lektüre der einzelnen Textstellen aus den Augen verlieren. Wie bereits gezeigt wurde, gibt es viele Verfahren, die die Verständlichkeit auf den Ebenen von Morphologie, Lexik, Syntax und Semantik erhöhen sollen. Auf der Textebene wird die Verständlichkeit dadurch aber wiederum beeinträchtigt (vgl. Bredel \& Maaß 2016: 481; ausführlich zur Textebene Bredel \& Maaß 2016: 481-513). Als erster Punkt sei an dieser Stelle die Verwendung von Pronomina herausgegriffen (dazu bereits Radünzel 2017: 83-86). Für die LS gilt das Grundprinzip, Gleiches immer gleich zu bezeichnen, also Synonyme zu vermeiden. Dies wirkt sich dahin gehend aus, dass zur Wiederaufnahme keine Personalpronomina der 3. Person verwendet, sondern die entsprechenden Nomina wiederholt werden, da es für die Adressaten schwierig sein kann zu entschlüsseln, worauf sich die Pronomina beziehen (vgl. Maaß 2015: 134f.). ${ }^{6}$ Personalpronomina der 1. und 2. Person sind dagegen lizensiert. In den Texten kommen Pronomina der 3. Person vor, die man ersetzen könnte, z.B.: „Europejska Platforma Rzeczników Wlasnych Prawa sklada sie z organizacji rzeczników wlasnych praw z róznych / krajów w Europie. / Nazywamy ja EPSA, od jej angielskiej nazwy." (Rzecznictwo wlasne) $\rightarrow$ „My nazywamy tę platformę EPSA (od angielskiej nazwy tej platformy).“, ,"Rada Europy przygotowuje wazne rekomendacje dla wszystkich krajów Europy. / To nie sa prawa, ale wiele krajów je stosuje.“ (Prawa) $\rightarrow$ „Rada Europy przygotowuje ważne zalecenia dla wszystkich krajów Europy. / To nie są prawa. / Ale wiele krajów stosuje te zalecenia." Abweichend vom Deutschen kann im Polnischen das Pronomen in Subjektposition fehlen, wofür man im Textkorpus einige Belege findet. Untersuchungen müssten zeigen, ob dies den Rezipienten von LS-Texten das Verständnis erschwert. Unabhängig vom Ergebnis solcher Untersuchungen könnte man in Übereinstimmung mit dem Redundanzprinzip die Nomina einfügen, für die die (fehlenden) Pronomina stehen, z.B.: „Projekt zawsze zajmuje sie jednym, konkretnym tematem. / Ma swój poczatek i koniec." (Projekty) $\rightarrow$ „Projekt zawsze zajmuje się jednym konkretnym tematem. / Każdy projekt ma swój początek i koniec.“

Ein weiteres generelles Merkmal von LS-Texten ist die direkte Leseransprache. Sie fungiert, ebenso wie die oben schon angesprochene Vermeidung unper-

\footnotetext{
${ }^{6}$ Bei der Wiederholung von Nomina in Subjektposition in Sätzen mit gleicher Satzstellung ist allerdings das Phänomen der „repeated name penalty“ zu berücksichtigen (vgl. Bock \& Lange 2017: 267).
} 
sönlicher Konstruktionen, als Mittel zur Realisierung der Orientierungsfunktion (Bredel \& Maaß 2016: 506f.). Im Zusammenhang mit der direkten Leseransprache stellt sich die Frage nach der angemessenen Anrede. Die deutschen Regelwerke fordern einhellig das Siezen der Adressatenschaft (ausgenommen, man schreibt für Personen, die man auch sonst duzt, z.B. Mitglieder des eigenen Vereins o.Ä.) (BMAS: 48; IE: 11; ILSMH: 13; NLS: 19; Maaß 2015: 81, 136, 182). Dies ist absolut nachvollziehbar, da Menschen mit geistigen oder anderen Behinderungen keinesfalls wie unmündige Kinder behandelt werden sollten. Die deutsche Anrede mit "Sie“ ist hier üblich und unproblematisch. Anders verhält es sich im Polnischen (zu dieser Frage schon Radünzel 2017: 85). Dort gibt es insofern ein Problem, als die Distanzanrede an eine Einzelperson die Unterscheidung zwischen einem Mann und einer Frau erfordert („pan“ vs. „pani“). Richtet sich der Text an einen unbestimmten Adressaten, kann man zu dessen Geschlecht nichts aussagen. Direktive und andere Sprechakte, die für beliebige Adressaten formuliert sind, weisen im Polnischen verschiedene sprachliche Mittel auf. Hierzu gehört beispielsweise die nichtdistanzierte Form in Aufforderungen wie "Chroń swój komputer", in denen man im Deutschen die Distanzanrede („,So schützen Sie Ihren Computer" ) verwendet (Eaziński 2006: 118, 2007a: 32, 2007b: 132; generell Łaziński 2006: 117-131; Łaziński 2007a, 2007b). Angesichts dessen wäre eine Verwendung der 2. Person Singular in Erwägung zu ziehen, die dann nicht als unpassende Vertraulichkeit bzw. Bevormundung der Zielgruppen der LS bewertet werden könnte (so bereits die Empfehlung in Radünzel 2017: 85 unter Bezug auf die Arbeiten Łazińskis). Dies ist im Textmaterial der Fall. Hier werden sowohl Imperativformen und andere Verbformen der 2. Person Singular als auch das entsprechende Personal- und Possessivpronomen verwendet, z.B.: "Dowiedz sie wiecej o EPSA!“ (Rzecznictwo wlasne), „Na pewno znajdziesz wsród nich wydarzenia interesujace i dostepne dla Ciebie." (Zaangazuj sie), „Cenimy twoje opinie." (Zaangazuj sie).

\section{ZUSAMMENFASSUNG UND AUSBLICK}

Resümierend sei angemerkt, dass die eingesehenen Texte der Organisation "Inclusion Europe“ aufgrund ihrer übersichtlichen Struktur und ihrer vereinfachten sprachlichen Gestaltung durchaus geeignet sind, Personen, die aus verschiedenen Gründen Schwierigkeiten mit der Lektüre polnischer standardsprachlicher Texte haben, Informationen über den Verein zu vermitteln. Sie entsprechen jedoch in mancherlei Hinsicht nicht den Regeln, die von „Inclusion Europe" selbst aufgestellt worden sind. Wie deutlich geworden ist, können die sprachwissenschaftlichen Werke, die in jüngster Zeit zur LS im Deutschen er- 
schienen sind, durchaus Anhaltspunkte für das Polnische bieten. So konnten Sätze in vielen Fällen nach dem Vorbild des Deutschen umformuliert werden. An anderen Stellen hat sich aber klar gezeigt, dass wegen der Charakteristika des Polnischen nur auf der Grundlage sprachspezifischer Forschungen entschieden werden könnte, ob bestimmte Erscheinungen als Verständnishürden anzusehen sind und welche Lösungen denkbar wären. Hierfür müssten insbesondere empirische Untersuchungen mit Angehörigen der Zielgruppen der LS durchgeführt werden. Wie eingangs bereits angekündigt, hat dieser Artikel daher mehr Fragen aufgeworfen als Lösungen präsentiert. Wie aus den zu Beginn zitierten Angaben auf der Internetseite der polnischen Regierung ersichtlich ist, hat man das Problem verständlicher Informationen für alle durchaus erkannt. Linguistisch fundierte Vorschläge zur Ausarbeitung einer LS im Polnischen könnten hierzu einen Beitrag leisten.

\section{LITERATURVERZEICHNIS}

Bock, B. M. (2014). „Leichte Sprache“: Abgrenzung, Beschreibung und Problemstellungen aus Sicht der Linguistik. In: S. J. Jekat / H. E. Jüngst / K. Schubert / C. Villiger (Hrsg.). Sprache barrierefrei gestalten: Perspektiven aus der Angewandten Linguistik (S. 17-51). Berlin: Frank \& Timme.

Bock, B. M. / Lange, D. (2017). Empirische Untersuchungen zu Satz- und Textverstehen bei Menschen mit geistiger Behinderung und funktionalen Analphabeten. In: B. M. Bock / U. Fix / D. Lange (Hrsg.). „Leichte Sprache“ im Spiegel theoretischer und angewandter Forschung (S. 253-274). Berlin: Frank \& Timme.

Bredel, U. / Lang, K. / Maaß, Ch. (2016). Zur empirischen Überprüfbarkeit von Leichte-Sprache-Regeln am Beispiel der Negation. In: N. Mälzer (Hrsg.). Barrierefreie Kommunikation - Perspektiven aus Theorie und Praxis (S. 95-115). Berlin: Frank \& Timme.

Bredel, U. / Maaß, Ch. (2016). Duden Leichte Sprache: Theoretische Grundlagen. Orientierung für die Praxis. Berlin: Duden-Verlag.

Bredel, U. / Maaß, Ch. (2017). Wortverstehen durch Wortgliederung - Bindestrich und Mediopunkt in Leichter Sprache. In: B. M. Bock / U. Fix / D. Lange (Hrsg.). „Leichte Sprache“ im Spiegel theoretischer und angewandter Forschung (S. 211-228). Berlin: Frank \& Timme.

Bundesministerium für Arbeit und Soziales (Hrsg.) (2014). Leichte Sprache: Ein Ratgeber. Berlin.

Europäische Vereinigung der ILSMH (Hrsg.) (1998). Sag es einfach! Europäische Richtlinien für die Erstellung von leicht lesbaren Informationen für Menschen mit geistiger Behinderung für Autoren, Herausgeber, Informationsdienste, Übersetzer und andere interessierte Personen. Brüssel. http:// www.webforall.info/wp-content/uploads/2012/12/EURichtlinie_sag_es_einfach.pdf [Zugriff am: 10.11.2014].

Gesetz zur Gleichstellung behinderter Menschen (Behindertengleichstellungsgesetz - BGG) vom 27.4.2002, http://www.gesetze-im-internet.de/bundesrecht/bgg/gesamt.pdf [Zugriff am: 12.9.2015].

Gesetz zur Weiterentwicklung des Behindertengleichstellungsrechts vom 19.7.2016. In: Bundesgesetzblatt Jahrgang 2016 Teil I Nr. 36. Ausgegeben zu Bonn am 26.7.2016, 1757-1763.

Gross, S. (2015). Regeln und Standards für leicht verständliche Sprache: Ein Rundblick. In: K. Candussi / W. Fröhlich (Hrsg.). Leicht Lesen: Der Schlüssel zur Welt (S. 81-105). Wien / Köln / Weimar: Böhlau Verlag. 
Heine, A. (2017). Deutsch als Fremd- und Zweitsprache - eine besondere Form Leichter Sprache? Überlegungen aus der Perspektive des Faches DaF / DaZ. In: B. M. Bock / U. Fix / D. Lange (Hrsg.). "Leichte Sprache“ im Spiegel theoretischer und angewandter Forschung (S. 401-414). Berlin: Frank \& Timme.

Inclusion Europe (Hrsg.) (2009). Informationen für alle: Europäische Regeln, wie man Informationen leicht lesbar und leicht verständlich macht. Brüssel. http:/ / bidok.uibk.ac.at/download/inclusion-europe.pdf [Zugriff am: 10.11.2014].

Just, C. S. (2017). Vorgaben zu Leichter Sprache im Deutschen und Möglichkeiten ihrer Übertragung auf das Russische. Kiel: Masterarbeit im Fach Migration und Diversität der Philosophischen Fakultät der Christian-Albrechts-Universität zu Kiel.

Kilian, J. (2017). „Leichte Sprache“, Bildungssprache und Wortschatz - Zur sprach- und fachdidaktischen Wertigkeit der Regelkonzepte für „leichte Wörter“. In: B. M. Bock / U. Fix / D. Lange (Hrsg.). "Leichte Sprache“ im Spiegel theoretischer und angewandter Forschung (S. 189-209). Berlin: Frank \& Timme.

Lasch, A. (2017). Zum Verständnis morphosyntaktischer Merkmale in der funktionalen Varietät "Leichte Sprache“. In: B. M. Bock / U. Fix / D. Lange (Hrsg.). „Leichte Sprache“ im Spiegel theoretischer und angewandter Forschung (S. 275-299). Berlin: Frank \& Timme.

Łaziński, M. (2006). O panach i paniach: Polskie rzeczowniki tytularne i ich asymetria rodzajowo-ptciowa. Warszawa: Wydawnictwo Naukowe PWN.

Łaziński, M. (2007a). Między tytułomanią a poufałością: Formy bezdystansowe w polskich komunikatach publicznych. Seminaria Naukowe Wroctawskiego Towarzystwa Naukowego, 57 (6), 31-34.

Łaziński, M. (2007b). Między tytułomanią a poufałością: Polskie dyrektywy do nieokreślonego adresata na tle słowiańskim. In: Z. Rudnik-Karwatowa (red.). Z polskich studiów slawistycznych. Seria XI. Jezzykoznawstwo. Prace na XIV Międzynarodowy Kongres Slawistów w Ochrydzie 2008 (S. 129-137). Warszawa: Polska Akademia Nauk, Komitet Słowianoznawstwa.

Maaß, Ch. (2015). Leichte Sprache: Das Regelbuch. Berlin: Lit Verlag.

Maaß, Ch. / Rink, I. / Zehrer, Ch. (2014). Leichte Sprache in der Sprach- und Übersetzungswissenschaft. In: S. J. Jekat / H. E. Jüngst / K. Schubert / C. Villiger (Hrsg.). Sprache barrierefrei gestalten: Perspektiven aus der Angewandten Linguistik (S. 53-85). Berlin: Frank \& Timme.

Netzwerk Leichte Sprache (Hrsg.). Die Regeln für Leichte Sprache. http:/ / www.leichtesprache.org/ downloads/Regeln\%20fuer\%20Leichte\%20Sprache.pdf [Zugriff am: 10.11.2014].

Oomen-Welke, I. (2015). Leichte Sprache, Einfache Sprache und Deutsch als Zweitsprache. Didaktik Deutsch, 38, 24-32.

Przybyła-Wilkin, A. (2016). Easy-to-read in English, German and Polish. In: N. Mälzer (Hrsg.). Barrierefreie Kommunikation - Perspektiven aus Theorie und Praxis (S. 135-150). Berlin: Frank \& Timme.

Radünzel, C. (2017). Leichte Sprache: Eine linguistische Betrachtung eines neuen sprachlichen Phänomens auf der Grundlage polnischer und deutscher Beispieltexte. Zeitschrift für Slawistik, $62(1), 48-94$.

Radünzel, C. (2018). Dostupnyj jazyk: Istorija i charakteristika novoj jazykovoj raznovidnosti. In: S. Kempgen / M. Wingender / L. Udolph (Hrsg.). Deutsche Beiträge zum 16. Internationalen Slavistenkongress Belgrad 2018 (S. 269-278). Wiesbaden: Harrassowitz Verlag.

Riegert, J. / Musenberg, O. (2017). Zur didaktischen Bedeutung Leichter Sprache im inklusiven Unterricht. In: B. M. Bock / U. Fix / D. Lange (Hrsg.). "Leichte Sprache“ im Spiegel theoretischer und angewandter Forschung (S. 387-399). Berlin: Frank \& Timme.

Rink, I. (2014). Barrierefreie Kommunikation als Nachteilsausgleich (21.5.2014). In: Ch. Maaß / I. Rink / Ch. Zehrer. Leichte Sprache. Forschungsstelle Leichte Sprache. http://www.uni-hil- 
desheim.de/fb3/institute/institut-fuer-uebersetzungswiss-fachkommunikation/forschung/ leichtesprache/forschung/ [Zugriff am: 14.6.2015].

Die UN-Behindertenrechtskonvention: Übereinkommen über die Rechte von Menschen mit Behinderungen. https://www.behindertenbeauftragter.de/SharedDocs/Publikationen/DE/Broschuere_UNKonvention_KK.pdf?_blob=publicationFile [Zugriff am: 12.9.2015].

Usova, O. (2015). Leichte Sprache im Übersetzungskontext: Dargestellt an deutschen und russischen Textbeispielen zum Thema Barrierefreiheit. Dresden: Masterarbeit im Studiengang Europäische Sprachen, Institut für Slavistik der Technischen Universität Dresden.

Usova, O. (2017). Leichte Sprache in Russland. In: B. M. Bock / U. Fix / D. Lange (Hrsg.). „Leichte Sprache" im Spiegel theoretischer und angewandter Forschung (S. 457-461). Berlin: Frank \& Timme. Verordnung zur Schaffung barrierefreier Informationstechnik nach dem Behindertengleichstellungsgesetz (Barrierefreie-Informationstechnik-Verordnung - BITV 2.0) vom 12.9.2011 (BGBI. I S. 1843). http:/ / www.gesetze-im-internet.de/bundesrecht/bitv_2_0/gesamt.pdf [Zugriff am: 14.6.2015].

Zurstrassen, B. (2017). Leichte Sprache - eine Sprache der Chancengleichheit? In: B. M. Bock / U. Fix / D. Lange (Hrsg.). "Leichte Sprache“ im Spiegel theoretischer und angewandter Forschung (S. 53-69). Berlin: Frank \& Timme.

\section{QUELLEN}

Inclusion Europe (Hrsg.) (2005). Działalność Organizacji Narodów Zjednoczonych. o.O.

Inclusion Europe (Hrsg.) (2011). Rekomendacje dla polityki łatwiej dostępnych wyborów w Europie: Stworzone w ramach projektu „Dostosowanie Różnorodności do Aktywnego Udziału w Wyborach do Parlamentu Europejskiego" w maju 2011 roku. o.O.

Inclusion Europe (Hrsg.) (2011). Rekomendacje dla polityki łatwiej dostępnych wyborów w Europie: Wersja tatwa do odczytania. o.O.

Internetseite der Organisation „Inclusion Europe“, Informationen in polnischer Sprache. http:/ / inclusion-europe.eu/?page_id=83\&lang=pl [Zugriff am: 8.10.2016].

Internetseite der polnischen Regierung. www.premier.gov.pl [Zugriff am: 3.11.2019].

Internetseite des polnischen Parlaments. www.sejm.pl [Zugriff am: 3.11.2019].

Internetseite des Präsidenten der Republik Polen. www.prezydent.pl [Zugriff am: 3.11.2019].

Received: 9.01.2020; revised: 7.03.2020

CLAUDIA RADÜNZEL

Christian-Albrechts-Universität zu Kiel

c.raduenzel@email.uni-kiel.de

ORCID: 0000-0002-3406-6871 
